\title{
OSCILLATIONS IN LINEAR DIFFERENCE EQUATIONS WITH VARIABLE COEFFICIENTS ${ }^{1}$
}

\author{
CH. G. PHILOS and I.K. PURNARAS \\ Department of Mathematics \\ University of Ioannina \\ P.O. Box 1186 \\ 45110 Ioannina, GREECE
}

\begin{abstract}
A class of linear difference equations with variable coefficients is considered. Sufficient conditions and necessary conditions for the oscillation of the solutions are established. In the special cases where the coefficients are constant or periodic the conditions become both necessary and sufficient.
\end{abstract}

Key words: Linear difference equation, oscillation.

AMS (MOS) subject classification: Primary 39A10.

\section{INTRODUCTION}

Recently there has been a great deal of work on the oscillation of solutions of difference equations (see, for example, [2]-[5], [7]-[10], [14]-[16], [18], [19], and [21]).

Within the past two decades, the study of difference equations has acquired a new significance. This comes about, in large part, from the fact that difference equations appear as natural descriptions of observed evolution phenomena as well as in the study of discretization methods for differential equations. Furthermore, the theory of difference equations is rapidly gaining attention because of its use in such fields as numerical analysis, control theory, finite mathematics and computer science; in particular, because of the successful use in recent years of computers to solve difficult problems arising in applications, the connection between the theory of difference equations and computer science has become more important.

For a systematic treatment of the theory of difference equations and its applications to numerical analysis, the reader is referred to the recent book by Lakshmikantham and Trigiante [12]. Chapter 7 of this book is devoted to some applications of difference equations to many fields such as economics, chemistry, population dynamics and queueing theory. Some discrete models in

\footnotetext{
${ }^{1}$ Received: December, 1990. Revised: February, 1991.
} 
population dynamics have appeared in [6].

Consider the linear difference equation

$$
A_{n+1}-A_{n}+\sum_{k=0}^{m} p_{k}(n) A_{n-\ell_{k}}=0
$$

where $m$ is a positive integer, $\left(p_{k}(n)\right)_{n \geq 0}(k=0,1, \ldots, m)$ are sequences of nonnegative numbers, and $\ell_{k}(k=0,1, \ldots, m)$ are integers such that $0=\ell_{0}<\ell_{1}<\ldots<\ell_{m}$. The sequences $\left(p_{k}(n)\right)_{n \geq 0}(k=1, \ldots, m)$ are (supposed to be) not identically zero.

Let $n_{0}$ be a nonnegative integer and set

$$
\mathbf{N}_{n_{0}}=\left\{n_{0}, n_{0}+1, \ldots\right\}
$$

By a solution on $N_{n_{0}}$ of the difference equation $(E)$ we mean a sequence $\left(A_{n}\right)_{n \geq n_{0}}-\ell_{m}$ which satisfies $(E)$ for all $n \geq n_{0}$. As usual, a solution of $(E)$ is said to be nonoscillatory if it is either eventually positive or eventually negative. Otherwise, the solution is said to be oscillatory. A solution $\left(A_{n}\right)_{n \geq n_{0}}-\ell_{m}$ on $N_{n_{0}}$ of $(E)$ is called positive if $A_{n}>0$ for all $n \geq n_{0}-\ell_{m}$.

Let us consider the linear difference equation with constant coefficients

$$
A_{n+1}-A_{n}+\sum_{k=0}^{m} P_{k} A_{n-\ell_{k}}=0
$$

where $P_{k}(k=0,1, \ldots, m)$ are real numbers with $P_{0} \geq 0, P_{1}>0, \ldots, P_{m}>0$. This equation is a special case of the difference equation $(E)$. For the autonomous difference equation $\left(E_{0}\right)$ the following "if and only if" oscillation criterion is known (see [4], [9], [14]), which is the discrete analogue of a result due to Tramov [22] (see also [1], [11]) concerning the oscillation of first order linear delay differential equations with constant coefficients.

Theorem A: A necessary and sufficient condition for the oscillation of all solutions of the difference equation $\left(E_{0}\right)$ is that its characteristic equation

$$
F_{0}(\lambda) \equiv \lambda-1+\sum_{k=0}^{m} P_{k} \lambda^{-\ell_{k}}=0
$$

has no roots in $(0,1)$.

Next, let us consider another special case (which includes the previous one), i.e., the case where the coefficients in $(E)$ are periodic sequences with a common period and the integers $\ell_{k}(k=1, \ldots, m)$ are multiples of this period. In this case, the oscillation of the solutions of the difference equation $(E)$ is described by the following result due to Philos [18], which is the discrete analogue of an oscillation criterion of the same author [17] for first order linear delay differential 
equations with periodic coefficients.

Theorem B: Assume that $\left(p_{k}(n)\right)_{n \geq 0}(k=0,1, \ldots, m)$ are periodic sequences with a common period $L$ (where $L$ is a positive integer) and that there exist positive integers $v_{1}, \ldots, v_{m}$ such that

$$
\ell_{1}=v_{1} L, \ldots, \ell_{m}=v_{m} L
$$

Introduce the equation

$$
F(\lambda) \equiv \lambda^{L}-\prod_{r=0}^{L-1}\left(1-\sum_{k=0}^{m} p_{k}(r) \lambda^{-\ell_{k}}\right)=0,
$$

which is associated with $(E)$. Then we have:

$A$ necessary condition for the oscillation of all solutions of $(E)$ is that there is no root $\lambda_{0}$ in $(0,1)$ of $(*)$ with the property: If $L>1$, then

$$
\sum_{k=0}^{m} p_{k}(r) \lambda_{0}^{-\ell_{k}}<1 \quad(r=1, \ldots, L-1)
$$

(ii) A sufficient condition for the oscillation of all solutions of $(E)$ is that $(*)$ has no roots in $(0,1)$.

It is easy to see that Theorem $A$ can be obtained from Theorem $B$ for $L=1$.

Our purpose in this paper is to examine the oscillation of the solutions of the general difference equation $(E)$ in which the coefficients are variable (and not necessarily periodic). More precisely, our aim is to establish sufficient conditions for the oscillation of all solutions of $(E)$ and also conditions under which $(E)$ has at least one nonoscillatory solution. In the special case of the difference equation $\left(E_{0}\right)$ in which the coefficients are constant, our results lead to Theorem $A$. Also, Theorem $B$ can be obtained from the results of this paper by applying them to the special case where the coefficients are periodic with a common period and $\ell_{k}(k=1, \ldots, m)$ are multiples of this period. The application of our results to the above special cases of constant or periodic coefficients will be presented in Section 5 . The results of the present paper are motivated by Theorems $A$ and $B$ as well as by the recent results of Philos [20] concerning the oscillation of first order delay differential equations with variable coefficients (see also [13] for similar results).

\section{STATEMENT OF THE MAIN RESULTS}

Our main results are Theorems 1 and 2 below. Theorem 1 provides sufficient conditions for 
the oscillation of all solutions of the difference equation $(E)$. Conditions under which $(E)$ has at least one nonoscillatory solution are established by Theorem 2.

Theorem 1: Let $L$ and $v_{k}(k=1, \ldots, m)$ be positive integers such that

$$
\ell_{1}=v_{1} L, \ldots, \ell_{m}=v_{m} L
$$

Suppose that

$$
\lim _{n \rightarrow \infty} \inf \sum_{i=0}^{\ell_{1}} \sum_{k=1}^{m} p_{k}\left(i+n-\ell_{1}\right)>0
$$

Suppose also that, for some integer $\nu_{0} \geq \ell_{m}$,

$$
\sup _{n \geq v_{0}} \max _{1 \leq k \leq m} \max _{1 \leq s \leq v_{k}} \prod_{r=(s-1) L}^{s L-1}\left(1-\sum_{j=0}^{m} p_{j}\left(r+n-\ell_{k}\right)\right)<1 .
$$

Moreover, assume that, for every $\lambda \in(0,1)$, there exists an integer $\nu \geq \ell_{m}$ such that

$$
\lambda^{L}-\sup _{n \geq \nu_{0}} \max _{1 \leq k \leq m} \max _{1 \leq s \leq v_{k}} \prod_{r=(s-1) L}^{s L-1}\left(1-\sum_{j=0}^{m} p_{j}\left(r+n-\ell_{k}\right) \lambda^{-\ell_{j}}\right)>0 .
$$

Then all solutions of the difference equation $(E)$ are oscillatory.

Theorem 2: Assume that there exists a $\lambda \in(0,1)$ and an integer $n_{0} \geq 1+\ell_{m}$ such that

$$
\begin{aligned}
& \sum_{k=0}^{m} p_{k}(n) \lambda^{-\ell_{k}}<1 \text { for every } n \geq 0, \\
& \sum_{i=0}^{\ell_{1}-1} \sum_{k=1}^{m} p_{k}(n+i)>0 \text { for all } n \geq n_{0}
\end{aligned}
$$

and

$$
\sup _{n \geq n_{0}} \sum_{k=0}^{m} p_{k}(n)\left\{-\lambda^{-\ell_{k}}+\left[\prod_{r=0}^{\ell_{k}-1}\left(1-\sum_{j=0}^{m} p_{j}\left(r+n-\ell_{k}\right) \lambda^{-\ell_{j}}\right)\right]^{-1}\right\} \leq 0 .
$$

Then the difference equation $(E)$ has a positive solution $\left(A_{n}\right)_{n \geq n_{0}}-\ell_{m}$ on $N_{n_{0}}$ with $\lim _{n \rightarrow \infty} A_{n}=0$ and such that

$$
A_{n} \leq \prod_{r=0}^{n-1}\left(1-\sum_{k=0}^{m} p_{k}(r) \lambda^{-\ell_{k}}\right) \text { for every } n \geq n_{0}-\ell_{m}
$$

Note: In condition $\left(C_{2}\right)$, we have used the convention $\prod_{\rho}^{\sigma}=1$ when $\sigma<\rho$. This convention will also be used in the proof of Theorem 2 . 


\section{PROOF OF THEOREM 1}

Assume, for the sake of contradiction, that the difference equation $(E)$ has a nonoscillatory solution $\left(A_{n}\right)_{n \geq n_{0}}-\ell_{m}$ on $N_{n_{0}}$, where $n_{0}$ is a nonnegative integer. As the negative of a solution of $(E)$ is also a solution of the same equation, we may (and do) assume that $\left(A_{n}\right)_{n \geq n_{0}}-\ell_{m}$ is eventually positive. Furthermore, without loss of generality, we can suppose that $A_{n}>0$ for all $n \geq n_{0}-\ell_{m}$. Then from $(E)$ it follows that $A_{n+1}-A_{n} \leq 0$ for $n \geq n_{0}$ and so the sequence $\left(A_{n}\right)_{n} \geq n_{0}$ is decreasing.

For any $\lambda \in(0,1)$, we define

$$
c_{\lambda}(n)=1-\sum_{k=0}^{m} p_{k}(n) \lambda^{-\ell_{k}} \text { for } n \geq 0
$$

Furthermore, we consider the set

$$
\Lambda=\left\{\lambda \in(0,1): A_{n+1}-c_{\lambda}(n) A_{n} \leq 0 \text { for all large } n\right\} .
$$

The set $\Lambda$ is nonempty. In fact, by taking into account the decreasing character of the sequence $\left(A_{n}\right)_{n \geq n_{0}}$, from $(E)$ we obtain for $n \geq n_{0}+\ell_{m}$

$$
0=A_{n+1}-A_{n}+\sum_{j=0}^{m} p_{j}(n) A_{n-\ell_{j}} \geq A_{n+1}-A_{n}+\sum_{j=0}^{m} p_{j}(n) A_{n}
$$

and consequently

$$
A_{n+1}-\left(1-\sum_{j=0}^{m} p_{j}(n)\right) A_{n} \leq 0 \text { for every } n \geq n_{0}+\ell_{m} .
$$

This in particular implies that

$$
1-\sum_{j=0}^{m} p_{j}(n)>0 \text { for every } n \geq n_{0}+\ell_{m}
$$

Set

$$
\alpha=\left[\sup _{n \geq \nu_{0}} \max _{1 \leq k \leq m} \max _{1 \leq s \leq v_{k}} \prod_{r=(s-1) L}^{s L-1}\left(1-\sum_{j=0}^{m} p_{j}\left(r+n-\ell_{k}\right)\right)\right]^{1 / L}
$$

Because of (2) and assumption $\left(C_{0}\right)$, we have $0<\alpha<1$. If $i \in\{1,2, \ldots, m\}$, then from (1) we obtain for every integer $n$ with $n \geq \max \left\{\nu_{0}, n_{0}+2 \ell_{m}\right\}$

$$
\begin{gathered}
\frac{A_{n-\ell_{i}}}{A_{n}} \geq\left[\prod_{r=n-\ell_{i}}^{n-1}\left(1-\sum_{j=0}^{m} p_{j}(r)\right)\right]^{-1}=\left[\prod_{r=0}^{\ell_{i}-1}\left(1-\sum_{j=0}^{m} p_{j}\left(r+n-\ell_{i}\right)\right)\right]^{-1} \\
\quad=\left[\prod_{s=1}^{v_{i}} \prod_{r=(s-1) L}^{s L-1}\left(1-\sum_{j=0}^{m} p_{j}\left(r+n-\ell_{i}\right)\right)\right]^{-1}
\end{gathered}
$$




$$
\begin{aligned}
& \geq\left[\max _{1 \leq s \leq v_{i}} \prod_{r=(s-1) L}^{s L-1}\left(1-\sum_{j=0}^{m} p_{j}\left(r+n-\ell_{i}\right)\right)\right]^{-v_{i}} \\
& \geq\left[\sup _{n \geq v_{0}} \max _{1 \leq k \leq m} \max _{1 \leq s \leq v_{k}} \prod_{r=(s-1) L}^{s L-1}\left(1-\sum_{j=0}^{m} p_{j}\left(r+n-\ell_{k}\right)\right)\right]^{-v_{i}} \\
& =\left(\alpha^{L}\right)^{-v_{i}}=\alpha^{-v_{i} L}=\alpha^{-\ell_{i}} \text {. }
\end{aligned}
$$

Therefore,

$$
A_{n-\ell_{i}} \geq \alpha^{-\ell_{i}} A_{n} \text { for } n \geq \max \left\{\nu_{0}, n_{0}+2 \ell_{m}\right\} \quad(i=0,1, \ldots, m) .
$$

(The last inequality is obvious when $i=0$.) Thus, $(E)$ gives for every $n \geq \max \left\{\nu_{0}, n_{0}+2 \ell_{m}\right\}$

$$
\begin{gathered}
0=A_{n+1}-A_{n}+\sum_{i=0}^{m} p_{i}(n) A_{n-\ell_{i}} \geq A_{n+1}-\left(1-\sum_{i=0}^{m} p_{i}(n) \alpha^{-\ell_{i}}\right) A_{n} \\
=A_{n+1}-c_{\alpha}(n) A_{n} .
\end{gathered}
$$

This means that $\alpha \in \Lambda$ and so $\Lambda \neq \emptyset$. We can immediately see that, if $\lambda_{1} \in \Lambda$, then every number $\lambda_{2}$ with $\lambda_{1}<\lambda_{2}<1$ also belongs to $\Lambda$. So, $\Lambda$ is a subinterval of the interval $(0,1)$ and $\sup \Lambda=1$.

Next, we will establish that $\inf \Lambda>0$. By assumption $\left(H_{1}\right)$, there exist a constant $\beta>0$ and an integer $n_{1} \geq \ell_{1}$ so that

$$
\sum_{i=n-\ell_{1}}^{n} \quad \sum_{k=1}^{m} p_{k}(i)=\sum_{i=0}^{\ell_{1}} \quad \sum_{k=1}^{m} p_{k}\left(i+n-\ell_{1}\right) \geq \beta \text { for } n \geq n_{1} .
$$

Hence, for any $n \geq n_{1}$, we can choose an integer $n^{*} \equiv n^{*}(n)$ with $n-\ell_{1} \leq n^{*} \leq n$ such that

$$
\sum_{i=n-\ell_{1}}^{n^{*}} \sum_{k=1}^{m} p_{k}(i) \geq \frac{\beta}{2} \text { and } \sum_{i=n^{*}}^{n} \sum_{k=1}^{m} p_{k}(i) \geq \frac{\beta}{2} .
$$

Since the sequence $\left(A_{n}\right)_{n} \geq n_{0}$ is decreasing, from $(E)$ it follows that

$$
A_{n}-A_{n+1} \geq\left[\sum_{k=1}^{m} p_{k}(n)\right] A_{n-\ell_{1}} \text { for all } n \geq n_{0}+\ell_{m}
$$

Thus, by using again the fact that $\left(A_{n}\right)_{n} \geq n_{0}$ is a decreasing sequence, we obtain for $n \geq n_{0}+\ell_{m}+\ell_{1}$ 


$$
\begin{gathered}
A_{n^{*}}>A_{n^{*}}-A_{n+1}=\sum_{i=n^{*}}^{n}\left(A_{i}-A_{i+1}\right) \\
\geq \sum_{i=n^{*}}^{n}\left[\sum_{k=1}^{m} p_{k}(i)\right] A_{i-\ell_{1}} \geq\left[\sum_{i=n^{*}}^{n} \sum_{k=1}^{m} p_{k}(i)\right] A_{n-\ell_{1}} .
\end{gathered}
$$

Therefore, in view of (3), we derive

$$
A_{n *}>\frac{\beta}{2} A_{n-\ell_{1}} \text { for every } n \geq \max \left\{n_{1}, n_{0}+\ell_{m}+\ell_{1}\right\}
$$

Furthermore, consider an arbitrary element $\lambda$ of $\Lambda$. Then there exists an integer $n_{\lambda} \geq n_{0}$ such that

$$
A_{n+1}-\left(1-\sum_{k=0}^{m} p_{k}(n) \lambda^{-\ell_{k}}\right) A_{n} \leq 0 \text { for } n \geq n_{\lambda} \text {. }
$$

This gives

$$
A_{n}-A_{n+1} \geq\left[\sum_{k=1}^{m} p_{k}(n)\right] \lambda^{-\ell_{1}} A_{n} \text { for all } n \geq n_{\lambda}
$$

Hence, by the decreasing nature of the sequence $\left(A_{n}\right)_{n} \geq n_{0}$, we obtain for $n \geq n_{\lambda}+\ell_{1}$

$$
\begin{gathered}
A_{n-\ell_{1}}>A_{n-\ell_{1}}-A_{n^{*}+1}=\sum_{i=n-\ell_{1}}^{n^{*}}\left(A_{i}-A_{i+1}\right) \\
\geq\left\{\sum_{i=n-\ell_{1}}^{n^{*}}\left[\sum_{k=1}^{m} p_{k}(i)\right] A_{i}\right\} \lambda^{-\ell_{1}} \geq\left[\sum_{i=n-\ell_{1}}^{n^{*}} \sum_{k=1}^{m} p_{k}(i)\right] A_{n^{*}} \lambda^{-\ell_{1}} .
\end{gathered}
$$

So, by (3), we have

$$
A_{n-\ell_{1}}>\frac{\beta}{2} \lambda^{-\ell_{1}} A_{n^{*}} \text { for all } n \geq \max \left\{n_{1}, n_{\lambda}+\ell_{1}\right\}
$$

Combining (4) and (5), we conclude that

$$
1>\left(\frac{\beta}{2}\right)^{2} \lambda^{-\ell_{1}} \text { or } \lambda>\left(\frac{\beta}{2}\right)^{2 / \ell_{1}} \text {. }
$$

Thus, as $\lambda$ is an arbitrary element of $\Lambda$, we see that the positive number $\left(\frac{\beta}{2}\right)^{2 / \ell_{1}}$ is a lower bound of $\Lambda$ and so in $f \Lambda>0$.

Now, we set inf $\Lambda_{0}=\lambda_{0} \in(0,1)$ and we consider an arbitrary integer $\nu \geq \ell_{m}$. Moreover, we consider an arbitrary number $\theta$ in the interval $\left(\lambda_{0}, 1\right)$ and we put $\gamma=\lambda_{0} / \theta$. Then $\lambda_{0}<\gamma<1$ and consequently $\gamma \in \Lambda$. Hence, there exists an integer $n_{\gamma} \geq n_{0}$ so that

$$
A_{n+1}-c_{\gamma}(n) A_{n} \leq 0 \text { for all } n \geq n_{\gamma},
$$

i.e.

$$
A_{n+1}-\left(1-\sum_{j=0}^{m} p_{j}(n) \gamma^{-\ell_{j}}\right) A_{n} \leq 0 \text { for } n \geq n_{\gamma}
$$


This in particular implies that

$$
1-\sum_{j=0}^{m} p_{j}(n) \gamma^{-\ell_{j}}>0 \text { for every } n \geq n_{\gamma}
$$

Define

$$
q=\left[\sup _{n \geq \nu} \max _{1 \leq k \leq m} \max _{1 \leq s \leq v_{k}} \prod_{r=(s-1) L}^{s L-1}\left(1-\sum_{j=0}^{m} p_{j}\left(r+n-\ell_{k}\right) \gamma^{-\ell_{j}}\right)\right]^{1 / L} .
$$

By taking into account (7), we can see that $q$ is a positive number. Furthermore, if $i \in\{1,2, \ldots, m\}$, then from (6) it follows that for every $n \geq \max \left\{\nu, n_{\gamma}+\ell_{m}\right\}$

$$
\begin{aligned}
& \frac{A_{n-\ell_{i}}}{A_{n}} \geq\left[\prod_{r=n-\ell_{i}}^{n-1}\left(1-\sum_{j=0}^{m} p_{j}(r) \gamma^{-\ell_{j}}\right)\right]^{-1}=\left[\prod_{r=0}^{\ell_{i}-1}\left(1-\sum_{j=0}^{m} p_{j}\left(r+n-\ell_{i}\right) \gamma^{-\ell_{i}}\right)\right]^{-1} \\
& =\left[\prod_{s=1}^{v_{i}} \prod_{r=(s-1) L}^{s L-1}\left(1-\sum_{j=0}^{m} p_{j}\left(r+n-\ell_{i}\right) \gamma^{-\ell_{j}}\right)\right]^{-1} \\
& \geq\left[\max _{1 \leq s \leq v_{i}} \prod_{r=(s-1) L}^{s L-1}\left(1-\sum_{j=0}^{m} p_{j}\left(r+n-\ell_{i}\right) \gamma^{-\ell_{j}}\right)\right]^{-v_{i}} \\
& \geq\left[\sup _{n \geq \nu} \max _{1 \leq k \leq m} \max _{1 \leq s \leq v_{k}} \prod_{r=(s-1) L}^{s L-1}\left(1-\sum_{j=0}^{m} p_{j}\left(r+n-\ell_{k}\right) \gamma^{-\ell_{j}}\right)\right]^{-v_{i}} \\
& =\left(q^{L}\right)^{-v_{i}}=q^{-v_{i} L}=q^{-\ell_{i}} .
\end{aligned}
$$

So, we have

$$
A_{n-\ell_{i}} \geq q^{-\ell_{i}} A_{n} \text { for } n \geq \max \left\{\nu, n_{\gamma}+\ell_{m}\right\} \quad(i=0,1, \ldots, m) .
$$

(For $i=0$ the last inequality is obvious.) We now claim that

$$
q \geq \lambda_{0}
$$

If $q \geq 1$, then (9) is obvious. So, let us assume that $0<q<1$. By using (8), from (E) we obtain for $n \geq \max \left\{\nu, n_{\gamma}+\ell_{m}\right\}$

$$
\begin{gathered}
0=A_{n+1}-A_{n}+\sum_{i=0}^{m} p_{i}(n) A_{n-\ell_{i}} \geq A_{n}+1-\left(1-\sum_{i=0}^{m} p_{i}(n) q^{-\ell_{i}}\right) A_{n} \\
A_{n+1}-\left(1-\sum_{i=0}^{m} p_{i}(n) q^{\ell_{i}}\right) A_{n} \\
=A_{n+1}-c_{q}(n) A_{n},
\end{gathered}
$$


which means that $q \in \Lambda$ and consequently $q \geq \lambda_{0}$. Thus, (9) is true. Finally, (9) gives

$$
\sup _{n \geq \nu} \max _{1 \leq k \leq m} \max _{1 \leq s \leq v_{k}} \prod_{r=(s-1) L}^{s L-1}\left(1-\sum_{j=0}^{m} p_{j}\left(r+n-\ell_{k}\right) \gamma^{-\ell_{j}}\right) \geq \lambda_{0}^{L},
$$

namely

$$
\sup _{n \geq \nu} \max _{1 \leq k \leq m} \max _{1 \leq s \leq v_{k}} \prod_{r=(s-1) L}^{s L-1}\left(1-\sum_{j=0}^{m} p_{j}\left(r+n-\ell_{k}\right) \lambda_{0}-\ell_{j} \ell_{j}\right) \geq \lambda_{0}^{L} .
$$

Therefore, as $\theta \rightarrow 1-0$, we obtain

$$
\sup _{n \geq \nu} \max _{1 \leq k \leq m}^{\max } \max _{1 \leq s \leq v_{k}} \prod_{r=(s-1) L}^{s L-1}\left(1-\sum_{j=0}^{m} p_{j}\left(r+n-\ell_{k}\right) \lambda_{0}^{-\ell_{j}}\right) \geq \lambda_{0}^{L} .
$$

We have thus proved that there exists a number $\lambda_{0} \in(0,1)$ such that, for every integer $\nu \geq \ell_{m}$, (10) holds. This contradicts the assumption that, for every $\lambda \in(0,1)$, there exists an integer $\nu \geq \ell_{m}$ so that $\left(C_{1}\right)$ is satisfied. The proof of Theorem 1 is complete.

\section{PROOF OF THEOREM 2}

To prove Theorem 2 we will make use of the following result, which is interesting in its own right.

Lemma: Let $\left(B_{n}\right)_{n \geq n_{0}}-\ell_{m}$ be a positive solution on $N_{n_{0}}$, where $n_{0}$ is a nonnegative integer, of the difference inequality

$$
B_{n+1}-B_{n}+\sum_{k=0}^{m} p_{k}(n) B_{n-\ell_{k}} \leq 0 .
$$

Moreover, assume that condition $\left(\mathrm{H}_{2}\right)$ is satisfied.

Then there exists a positive solution $\left(A_{n}\right)_{n} \geq n_{0}-\ell_{m}$ on $N_{n_{0}}$ of the difference equation $(E)$ with $\lim _{n \rightarrow \infty} A_{n}=0$ and such that

$$
A_{n} \leq B_{n} \text { for every } n \geq n_{0}-\ell_{m}
$$

Note: If $n_{0}$ is a nonnegative integer, by a solution on $N_{n_{0}}$ of the difference inequality $(I)$ we mean a sequence $\left(B_{n}\right)_{n \geq n_{0}}-\ell_{m}$ which satisfies $(I)$ for all $n \geq n_{0}$. A solution $\left(B_{n}\right)_{n \geq n_{0}}-\ell_{m}$ on $N_{n_{0}}$ of $(I)$ is said to be positive if $B_{n}>0$ for every $n \geq n_{0}-\ell_{m}$. 
Proof of the Lemma: The method of proof is similar to that of Theorem 3 in [10] concerning a special case (see also Lemma in Section 3 of [16]).

From $(I)$ we obtain for $\nu \geq n \geq n_{0}$

$$
B_{n}>-\left(B_{\nu+1}-B_{n}\right)=-\sum_{j=n}^{\nu}\left(B_{j+1}-B_{j}\right) \geq \sum_{j=n}^{\nu} \sum_{k=0}^{m} p_{k}(j) B_{j-\ell_{k}}
$$

and consequently

$$
B_{n} \geq \sum_{j=n}^{\infty} \sum_{k=0}^{m} p_{k}(j) B_{j-\ell_{k}} \text { for all } n \geq n_{0}
$$

Consider the space $\mathcal{A}$ of all sequences $\left(A_{n}\right)_{n \geq n_{0}}-\ell_{m}$ satisfying

$$
A_{n}=B_{n} \text { for } n_{0}-\ell_{m} \leq n<n_{0} \text {, and } 0 \leq A_{n} \leq B_{n} \text { for } n \geq n_{0} .
$$

For any sequence $\left(A_{n}\right)_{n \geq n_{0}}-\ell_{m}$ in $\mathcal{A}$, we define

$$
S A_{n}=\left\{\begin{array}{c}
B_{n}, \text { if } n_{0}-\ell_{m} \leq n<n_{0} \\
\sum_{j=n}^{\infty} \sum_{k=0}^{m} p_{k}(j) A_{j-\ell_{k}}, \text { if } n \geq n_{0}
\end{array}\right.
$$

and, by using (11), we see that $\left(S A_{n}\right)_{n \geq n_{0}-\ell_{m}}$ is a sequence in the space $\mathcal{A}$. Thus, the above formula defines an operator $S: \mathcal{A} \rightarrow \mathcal{A}$. We can easily see that this operator is monotonic in the following sense: If $\left(A_{n}^{1}\right)_{n \geq n_{0}}-\ell_{m}$ and $\left(A_{n}^{2}\right)_{n \geq n_{0}}-\ell_{m}$ are two sequences in $\mathcal{A}$ such that

$$
A_{n}^{1} \leq A_{n}^{2} \text { for every } n \geq n_{0}-\ell_{m}
$$

then we also have

$$
S A_{n}^{1} \leq S A_{n}^{2} \text { for all } n \geq n_{0}-\ell_{m} .
$$

Now, we introduce the sequence $\left(\left(A_{n}^{r}\right)_{n \geq n_{0}}-\ell_{m}\right)_{r \geq 0}$ of points in $\mathcal{A}$ which is defined by

and

$$
A_{n}^{0}=B_{n} \text { for } n \geq n_{0}-\ell_{m}
$$

$$
A_{n}^{r}=S A_{n}^{r-1} \text { for } n \geq n_{0}-\ell_{m}(r=1,2, \ldots)
$$

It follows easily that

$$
A_{n}^{0} \geq A_{n}^{1} \geq A_{n}^{2} \geq \ldots \text { for } n \geq n_{0}-\ell_{m}
$$

and so we can define 


$$
A_{n}=\lim _{r \rightarrow \infty} A_{n}^{r} \text { for } n \geq n_{0}-\ell_{m}
$$

Then we observe that

$$
0 \leq A_{n} \leq B_{n} \text { for all } n \geq n_{0}-\ell_{m}
$$

Moreover, we can obtain

$$
A_{n}=\sum_{j=n}^{\infty} \sum_{k=0}^{m} p_{k}(j) A_{j-\ell_{k}} \text { for } n \geq n_{0}
$$

This gives

$$
\lim _{n \rightarrow \infty} A_{n}=0
$$

and

$$
A_{n+1}-A_{n}=-\sum_{k=0}^{m} p_{k}(n) A_{n-\ell_{k}} \text { for all } n \geq n_{0} .
$$

The last equation means that the sequence $\left(A_{n}\right)_{n \geq n_{0}}-\ell_{m}$ is a solution on $N_{n_{0}}$ of the difference equation $(E)$. We see that $A_{n}=B_{n}>0$ for $n_{0}-\ell_{m} \leq n<n_{0}$. It remains to show that $A_{n}$ is also positive for $n \geq n_{0}$. Assume, for the sake of contradiction, that $N \geq n_{0}$ is the first zero of $\left(A_{n}\right)_{n \geq n_{0}}-\ell_{m}$. That is,

$$
A_{n}>0 \text { for } n_{0}-\ell_{m} \leq n<N, \text { and } A_{N}=0 .
$$

Then, by using the hypothesis $\left(H_{2}\right)$, we obtain

$$
\begin{aligned}
& 0 \leq A_{N+\ell_{1}}=A_{N+\ell_{1}}-A_{N}=\sum_{\mu=N}^{N+\ell_{1}-1}\left(A_{\mu+1}-A_{\mu}\right) \\
& =-\sum_{\mu=N}^{N+\ell_{1}-1} \sum_{k=0}^{m} p_{k}(\mu) A_{\mu-\ell_{k}} \leq-\sum_{\mu=N}^{N+\ell_{1}-1} \sum_{k=1}^{m} p_{k}(\mu) A_{\mu-\ell_{k}} \\
& \leq-\left(\min _{N \leq \mu \leq N+\ell_{1}-1} \min _{1 \leq k \leq m} A_{\mu-\ell_{k}}\right) \sum_{\mu=N}^{N+\ell_{1}-1} \sum_{k=1}^{m} p_{k}(\mu) \\
& \leq-\left(\min _{N-\rho \leq N-1}^{\min } A_{\rho}\right) \sum_{i=0}^{\ell_{1}-1} \sum_{k=1}^{m} p_{k}(N+i)<0 .
\end{aligned}
$$

This is a contradiction and hence the proof of our lemma is complete.

We are now ready to give the proof of Theorem 2. Define

$$
B_{n}=\prod_{r=0}^{n-1} c_{\lambda}(r) \text { for } n \geq 1,
$$


where

$$
c_{\lambda}(n)=1-\sum_{k=0}^{m} p_{k}(n) \lambda^{-\ell_{k}} \text { for } n \geq 0 .
$$

By assumption $(H)$, we can see that

$$
B_{n}>0 \text { for every } n \geq 1 .
$$

Next, by using condition $\left(C_{2}\right)$, we obtain for every $n \geq n_{0}$

$$
\begin{gathered}
B_{n+1}-B_{n}+\sum_{k=0}^{m} p_{k}(n) B_{n-\ell_{k}}= \\
=\left[c_{\lambda}(n)-1\right] \prod_{r=0}^{n-1} c_{\lambda}(r)+\sum_{k=0}^{m} p_{k}(n) \prod_{r=0}^{n-\ell_{k}-1} c_{\lambda}(r) \\
=\left\{c_{\lambda}(n)-1+\sum_{k=0}^{m} p_{k}(n)\left[\prod_{r=n-\ell_{k}}^{n-1} c_{\lambda}(r)\right]^{-1}\right\}_{r=0}^{n-1} c_{\lambda}(r) \\
=\left\{-\sum_{k=0}^{m} p_{k}(n) \lambda^{-\ell_{k}}+\sum_{k=0}^{m} p_{k}(n)\left[\prod_{r=n-\ell_{k}}^{n-1}\left(1-\sum_{j=0}^{m} p_{j}(r) \lambda^{-\ell_{j}}\right)\right]^{-1}\right\}_{n} B_{n} \\
=B_{n} \sum_{k=0}^{m} p_{k}(n)\left\{-\lambda-\ell_{k}+\left[\prod_{r=n-\ell_{k}}^{n-1}\left(1-\sum_{j=0}^{m} p_{j}(r) \lambda^{-\ell_{j}}\right)\right]^{-1}\right\} \\
=B_{n} \sum_{k=0}^{m} p_{k}(n)\left\{-\lambda-\ell_{k}+\left[\prod_{r=0}^{\ell_{k}-1}\left(1-\sum_{j=0}^{m} p_{j}\left(r+n-\ell_{k}\right) \lambda^{-\ell_{j}}\right)\right]^{-1}\right\} \\
\leq B_{n} \begin{array}{c}
\sup _{n \geq n_{0}} \sum_{k=0}^{m} p_{k}(n)\left\{-\lambda^{-\ell_{k}}+\left[\prod_{r=0}^{\ell_{k}-1}\left(1-\sum_{j=0}^{m} p_{j}\left(r+n-\ell_{k}\right) \lambda^{-\ell_{j}}\right)\right]^{-1}\right\} \\
\leq 0 .
\end{array}
\end{gathered}
$$

Thus, the sequence $\left(B_{n}\right)_{n \geq n_{0}}-\ell_{m}$ is a positive solution on $N_{n_{0}}$ of the difference inequality (I). So, it suffices to apply our lemma to complete the proof of Theorem 2.

\section{APPLICATION OF THE MAIN RESULTS TO THE SPECLAL CASES OF CONSTANT OR PERIODIC COEFFICIENTS}

In this section, we shall apply our results to the special cases where the coefficients in the difference equation $(E)$ are constant or periodic. More precisely, we will show that Theorems $\mathrm{A}$ and 
B can be obtained from Theorems 1 and 2.

The case of constant coefficients: Consider the special case where the coefficients are constant, i.e., the case of the autonomous difference equation $\left(E_{0}\right)$.

Assume first that the characteristic equation $(*)_{0}$ of $\left(E_{0}\right)$ has no roots in $(0,1)$. Since $F_{0}(1)=\sum_{k=0}^{m} P_{k}>0$, we must have

$$
\lambda-1+\sum_{k=0}^{m} P_{k} \lambda^{-\ell_{k}}>0 \text { for all } \lambda \in(0,1)
$$

In order to apply here Theorem 1 , we choose $L=1, v_{k}=\ell_{k}(k=1, \ldots, m)$, and $\nu_{0}=\nu=\ell_{m}$. Then we can immediately see that conditions $\left(H_{1}\right)$ and $\left(C_{0}\right)$ hold by themselves. Moreover, one can verify that, for every $\lambda \in(0,1)$, condition $\left(C_{1}\right)$ is satisfied because of (12). Thus, Theorem 1 can be applied to guarantee the oscillation of all solutions of $\left(E_{0}\right)$.

Suppose, conversely, that $(*)_{0}$ has a root $\lambda \in(0,1)$. Then $\sum_{k=0}^{m} P_{k} \lambda^{-\ell_{k}}=1-\lambda<1$ and so $(H)$ is true. Choose $n_{0}=1+\ell_{m}$. We can see that $\left(H_{2}\right)$ holds by itself. Furthermore, assumption $\left(\mathrm{H}_{2}\right)$ takes the form

$$
\sum_{k=0}^{m} P_{k}\left[-\lambda^{-\ell_{k}}+\left(1-\sum_{j=0}^{m} P_{j} \lambda^{-\ell_{j}}\right)^{-\ell_{k}}\right] \leq 0
$$

This inequality is satisfied, since $\lambda \in(0,1)$ is a root of $(*)_{0}$. So, Theorem 2 ensures that $\left(E_{0}\right)$ has a nonoscillatory solution.

The case of periodic coefficients: Assume that the sequences $\left(p_{k}(n)\right)_{n \geq 0}(k=0,1, \ldots, m)$ are periodic with a common period $L$ (where $L$ is a positive integer) and that there exist positive integers $v_{k}(k=1, \ldots, m)$ such that

$$
\ell_{1}=v_{1} L, \ldots, \ell_{m}=v_{m} L
$$

Let there exist a root $\lambda \in(0,1)$ of the equation $(*)$ with the property: If $L>1$, then

$$
\sum_{k=0}^{m} p_{k}(r) \lambda^{-\ell_{k}}<1 \quad(r=1, \ldots, L-1)
$$

Then from (*) it follows that

$$
\sum_{k=0}^{m} p_{k}(r) \lambda^{-\ell_{k}}<1 \text { for all } r \in\{0,1, \ldots, L-1\}
$$

This means that $(H)$ holds, since the sequences $\left(p_{k}(n)\right)_{n \geq 0}(k=0,1, \ldots, m)$ are $L$-periodic. We now choose $n_{0}=1+\ell_{m}$. Since the sequences $\left(p_{k}(n)\right)_{n \geq 0}(k=1, \ldots, m)$ are $L$-periodic and not identically zero and $\ell_{1}=v_{1} L$, we can easily verify that condition $\left(H_{2}\right)$ is also satisfied. 
Furthermore, by taking into account the fact that $\left(p_{j}(n)\right)_{n \geq 0}(j=0,1, \ldots, m)$ are $L$-periodic sequences and that $\ell_{k}=v_{k} L(k=1, \ldots, m)$, we obtain for any $k \in\{1, \ldots, m\}$ and for every $n \geq n_{0}$

$$
\begin{gathered}
\prod_{r=0}^{\ell_{k}-1}\left(1-\sum_{j=0}^{m} p_{j}\left(r+n-\ell_{k}\right) \lambda^{-\ell_{j}}\right)=\prod_{r=n-\ell_{k}}^{n-1}\left(1-\sum_{j=0}^{m} p_{j}(r) \lambda^{-\ell_{j}}\right) \\
=\prod_{r=0}^{\ell_{k}-1}\left(1-\sum_{j=0}^{m} p_{j}(r) \lambda^{-\ell_{j}}\right)=\left[\prod_{r=0}^{L-1}\left(1-\sum_{j=0}^{m} p_{j}(r) \lambda^{-\ell_{j}}\right)\right]^{v_{k}} .
\end{gathered}
$$

Thus, condition $\left(C_{2}\right)$ becomes

$$
\sup _{n \geq n_{0}} \sum_{k=0}^{m} p_{k}(n)\left\{-\left(\lambda^{L}\right)^{-v_{k}}+\left[\prod_{r=0}^{L-1}\left(1-\sum_{j=0}^{m} p_{j}(r) \lambda^{-\ell_{j}}\right)\right]^{-v_{k}}\right\} \leq 0,
$$

which is true since $\lambda \in(0,1)$ is a root of $(*)$. So, by Theorem 2 , the difference equation $(E)$ admits a nonoscillatory solution.

Now, let us suppose that the equation $(*)$ has no roots in the interval $(0,1)$. Moreover, assume for the sake of contradiction that $(E)$ has at least one nonoscillatory solution. Then, as in the proof of Theorem 1 (cf. inequality (2)), we can verify that

$$
1-\sum_{k=0}^{m} p_{k}(n)>0 \text { for all large } n
$$

This means that

$$
1-\sum_{k=0}^{m} p_{k}(r)>0 \text { for } r=0,1, \ldots, L-1
$$

Furthermore, since the sequences $\left(p_{k}(n)\right)_{n \geq 0}(k=1, \ldots, m)$ are $L$-periodic and not identically zero, we can see that

$$
\prod_{r=0}^{L-1}\left(1-\sum_{k=0}^{m} p_{k}(r)\right)<1
$$

This gives $F(1)>0$ and so, as $F(\lambda)=0$ has no roots in $(0,1)$, we always have

$$
\lambda^{L}-\prod_{r=0}^{L-1}\left(1-\sum_{k=0}^{m} p_{k}(r) \lambda^{-\ell}\right)>0 \text { for all } \lambda \in(0,1) .
$$

We will apply Theorem 1 . Since the sequences $\left(p_{k}(n)\right)_{n \geq 0}(k=1, \ldots, m)$ are $L$-periodic and not identically zero, we have for every $n \geq \ell_{1}$

$$
\sum_{i=0}^{\ell_{1}} \sum_{k=1}^{m} p_{k}\left(i+n-\ell_{1}\right) \geq \sum_{i=0}^{\ell_{1}-1} \sum_{k=1}^{m} p_{k}\left(i+n-\ell_{1}\right)=\sum_{\mu=0}^{\ell_{1}-1} \sum_{k=1}^{m} p_{k}(\mu)>0
$$


and hence $\left(H_{1}\right)$ is fulfilled. Next, we choose $\nu_{0}=\nu=\ell_{m}$. By using again the $L$-periodicity of the sequences $\left(p_{j}(n)\right)_{n \geq 0}(j=0,1, \ldots, m)$ and the fact that $\ell_{k}=v_{k} L$, we obtain for all $n \geq \nu_{0}$, $k \in\{1, \ldots, m\}$ and $s \in\left\{1, \ldots, v_{k}\right\}$

$$
\begin{gathered}
\prod_{r=(s-1) L}^{s L-1}\left(1-\sum_{j=0}^{m} p_{j}\left(r+n-\ell_{k}\right)\right)=\prod_{r=0}^{L-1}\left(1-\sum_{j=0}^{m} p_{j}\left(r+n-\ell_{k}\right)\right) \\
=\prod_{r=n-\ell_{k}}^{n-\ell_{k}+L-1}\left(1-\sum_{j=0}^{m} p_{j}(r)\right)=\prod_{r=0}^{L-1}\left(1-\sum_{j=0}^{m} p_{j}(r)\right) .
\end{gathered}
$$

So, assumption $\left(C_{0}\right)$ holds because of (13). In a similar way, we can see that for every $\lambda \in(0,1)$

$$
\prod_{r=(s-1) L}^{s L-1}\left(1-\sum_{j=0}^{m} p_{j}\left(r+n-\ell_{k}\right) \lambda^{-\ell_{j}}\right)=\prod_{r=0}^{L-1}\left(1-\sum_{j=0}^{m} p_{j}(r) \lambda^{-\ell_{j}}\right) .
$$

for $n \geq \nu, k \in\{1, \ldots, m\}$ and $s \in\left\{1, \ldots, v_{k}\right\}$. Thus, in view of (14), condition $\left(C_{1}\right)$ is satisfied for each $\lambda \in(0,1)$. So, we can apply Theorem 1 to conclude that $(E)$ has no nonoscillatory solutions, a contradiction.

\section{A BRIEF DISCUSSION}

Concerning Theorem 1 it remains an open question to the authors if an analogous oscillation result can be obtained without the restriction that there exist positive integers $L$ and $v_{k}$ $(k=1, \ldots, m)$ so that $\ell_{1}=v_{1} L, \ldots, \ell_{m}=v_{m} L$. Furthermore, it is an open problem to extend the results of this paper to the more general case of difference equations of the form

$$
A_{n+1}-A_{n}+\sum_{k=0}^{m} p_{k}(n) A_{n-\ell_{k}(n)}=0
$$

where $\ell_{0}(n)=0$ for $n \geq 0$, and $\left(\ell_{k}(n)\right)_{n \geq 0}(k=1, \ldots, m)$ are sequences of positive integers such that

$$
\lim _{n \rightarrow \infty}\left(n-\ell_{k}(n)\right)=\infty \quad(k=1, \ldots, m)
$$

Equation $(E)$ is also called a delay difference equation. It will be the subject of a future work to present an analogous investigation with that of this paper for the advanced difference equation

$$
A_{n+1}-A_{n}-\sum_{k=0}^{m} p_{k}(n) A_{n+\ell_{k}}=0
$$

(see [15] for the case of advanced difference equations with constant coefficients). 


\section{REFERENCES}

[1] O. Arino, I. Györi, and A. Jawhari, "Oscillation criteria in delay equations", $J$. Differential Equations, 53 (1984), pp. 115-123.

[2] L.H. Erbe and B.G. Zhang, "Oscillation of discrete analogues of delay equations", Differential and Integral Equations, 2 (1989), pp. 300-309.

[3] I. Györi, "Sharp conditions for existence of nontrivial invariant cones of nonnegative initial values of difference equations", Appl. Math. Comput., 36 (1990), pp. 89-111.

[4] I. Györi and G. Ladas, "Linearized oscillations for equations with piecewise constant arguments", Differential and Integral Equations, 2 (1989), pp. 123-131.

[5] I. Györi, G. Ladas, and L. Pakula, "Conditions for oscillation of difference equations with applications to equations with piecewise constant arguments", SIAM J. Math. Anal., to appear.

[6] G. Karakostas, Ch.G. Philos, and Y.G. Sficas, "The dynamics of some discrete population models", Nonlinear Anal., to appear.

[7] G. Ladas, "Oscillations of equations with piecewise constant mixed arguments", Inter. Conference on Theory and Applications of Differential Equations, March 21-25, 1988, Ohio University, to be published by Marcel Dekker, Inc..

[8] G. Ladas, "Explicit conditions for the oscillation of difference equations", J. Math. Anal. Appl., 153 (1990), pp. 276-287.

[9] G. Ladas, Ch.G. Philos, and Y.G. Sficas, "Necessary and sufficient conditions for the oscillation of difference equations", Libertas Math., 9 (1989), pp. 121-125.

[10] G. Ladas, Ch.G. Philos, and Y.G. Sficas, "Sharp conditions for the oscillation of delay difference equations", J. Appl. Math. Simulation, 2 (1989), pp. 101-112.

[11] G. Ladas, Y.G. Sficas, and I.P. Stavroulakis, "Necessary and sufficient conditions for oscillations", Amer. Math. Monthly, 90 (1983), pp. 637-640; see also "Necessary and sufficient conditions for oscillations of higher order delay differential equations", Trans. Amer. Math. Soc., 285 (1984), pp. 81-90.

[12] V. Lakshmikantham and D. Trigiante, "Theory of Difference Equations: Numerical Methods and Applications", Academic Press, New York, 1988.

[13] V.A. Nadareishvili, "Oscillation and nonoscillation of solutions of first-order linear differential equations with deviating arguments", Differentsial'nye Uravneniya, 25 (1989), pp. 611-616 (Differential Equations, 25 (1989), pp. 412-417).

[14] E.G. Partheniadis, "Stability and oscillation of neutral delay differential equations with piecewise constant argument", Differential and Integral Equations, 1 (1988), 
pp. $459-472$.

[15] Ch.G. Philos, "Oscillations in certain difference equations", Utilitas Math., in press.

[16] Ch.G. Philos, “On oscillations of some difference equations”, Funkcial. Ekvac., to appear.

[17] Ch.G. Philos, "On the oscillation of differential equations with periodic coefficients", Proc. Amer. Math. Soc., in press.

[18] Ch.G. Philos, "Oscillations in difference equations with periodic coefficients", Arch. Math., to appear.

[19] Ch.G. Philos, "Oscillations in a nonautonomous delay logistic difference equation", Proc. Edinburgh Math. Soc. (2), to appear.

[20] Ch.G. Philos, "Oscillation for first order linear delay differential equations with variable coefficients", Funkcial. Ekvac., to appear.

[21] Ch.G. Philos and Y.G. Sficas, "Positive solutions of difference equations", Proc. Amer. Math. Soc., 108 (1990), pp. 107-115.

[22] M.I. Tramov, "Conditions for oscillatory solutions of first order differential equations with a delayed argument”, Izv. Vysš. Učebn. Zaved., Matematica, 19 (1975), pp. 92-96. 


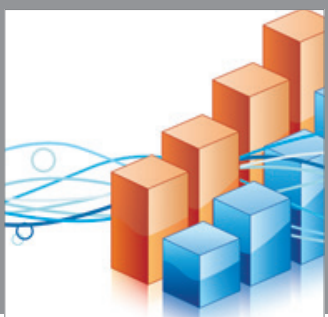

Advances in

Operations Research

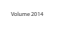

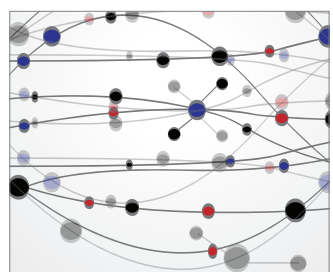

\section{The Scientific} World Journal
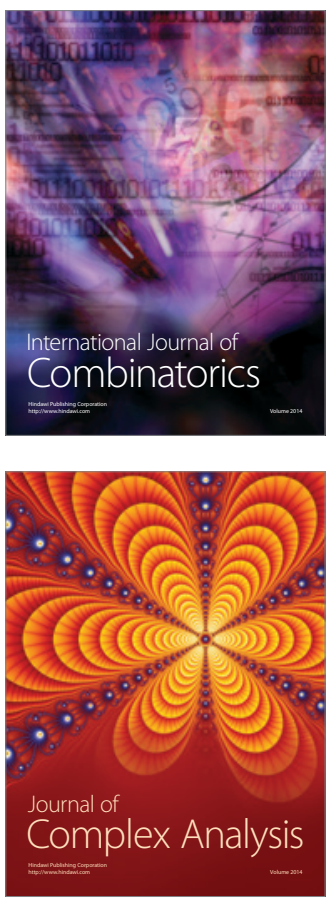

International Journal of

Mathematics and

Mathematical

Sciences
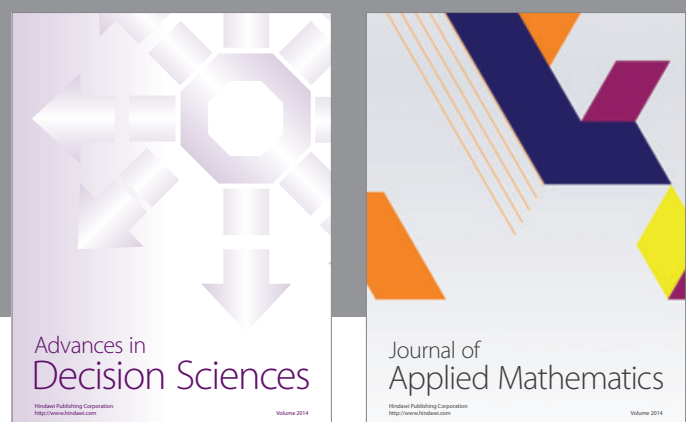

Journal of

Applied Mathematics
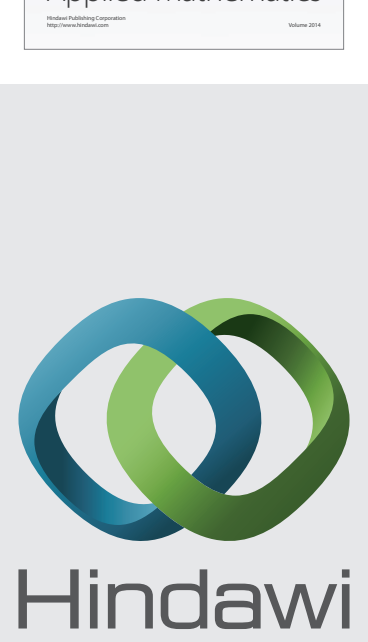

Submit your manuscripts at http://www.hindawi.com
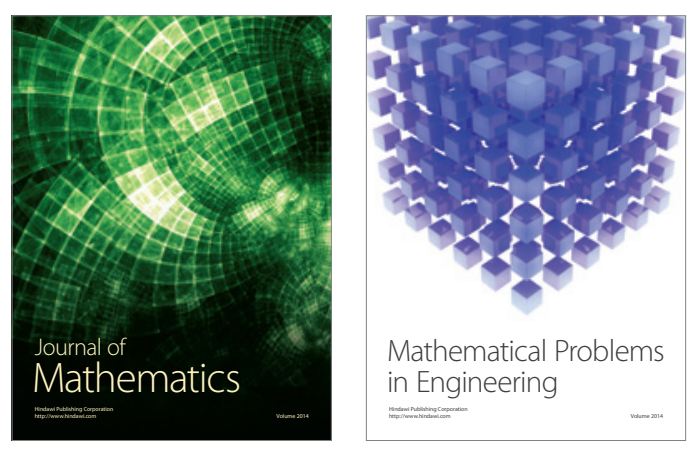

Mathematical Problems in Engineering
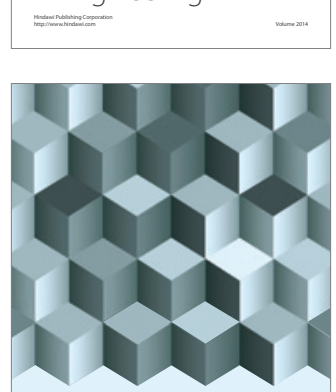

Journal of

Function Spaces
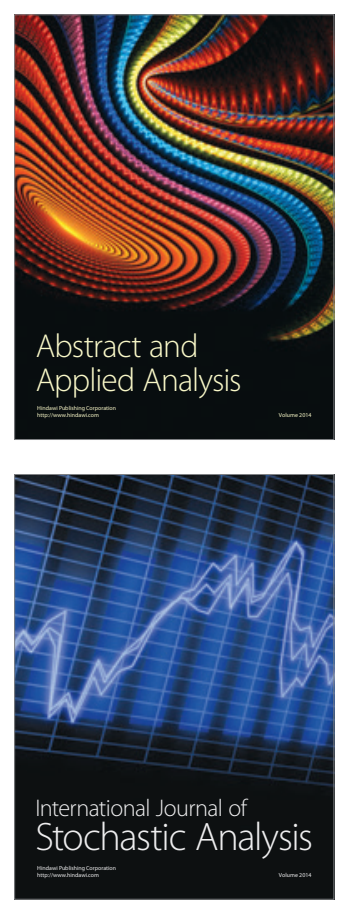

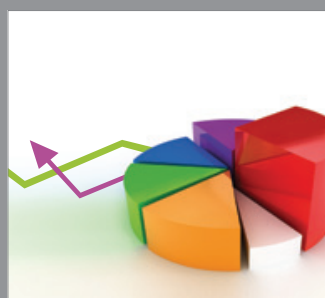

ournal of

Probability and Statistics

Promensencen
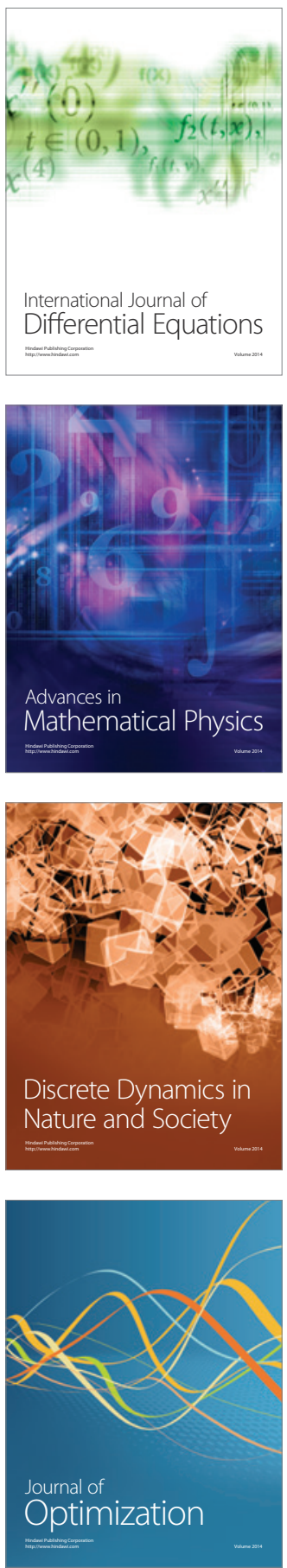